\title{
Engineering Polarons at a Metal Oxide Surface
}

\author{
C. M. Yim, ${ }^{1}$ M. B. Watkins, ${ }^{2}$ M. J. Wolf,${ }^{3,4}$ C. L. Pang, ${ }^{1}$ K. Hermansson, ${ }^{4}$ and G. Thornton ${ }^{1, *}$ \\ ${ }^{1}$ Department of Chemistry and London Centre for Nanotechnology, University College London, \\ 20 Gordon Street, London WC1H OAJ, United Kingdom \\ ${ }^{2}$ School of Mathematics and Physics, University of Lincoln, Brayford Pool, Lincoln LN6 7TS, United Kingdom \\ ${ }^{3}$ Department of Physics \& Astronomy and London Centre for Nanotechnology, University College London, \\ Gower Street, London WC1E 6BT, United Kingdom \\ ${ }^{4}$ Department of Chemistry, Angström Laboratory, Uppsala University, Box 538, S-751 21 Uppsala, Sweden
}

(Received 4 February 2016; revised manuscript received 4 August 2016; published 9 September 2016)

\begin{abstract}
Polarons in metal oxides are important in processes such as catalysis, high temperature superconductivity, and dielectric breakdown in nanoscale electronics. Here, we study the behavior of electron small polarons associated with oxygen vacancies at rutile $\mathrm{TiO}_{2}(110)$, using a combination of low temperature scanning tunneling microscopy (STM), density functional theory, and classical molecular dynamics calculations. We find that the electrons are symmetrically distributed around isolated vacancies at $78 \mathrm{~K}$, but as the temperature is reduced, their distributions become increasingly asymmetric, confirming their polaronic nature. By manipulating isolated vacancies with the STM tip, we show that particular configurations of polarons are preferred for given locations of the vacancies, which we ascribe to small residual electric fields in the surface. We also form a series of vacancy complexes and manipulate the Ti ions surrounding them, both of which change the associated electronic distributions. Thus, we demonstrate that the configurations of polarons can be engineered, paving the way for the construction of conductive pathways relevant to resistive switching devices.
\end{abstract}

DOI: 10.1103/PhysRevLett.117.116402

When an electronic charge carrier is introduced into a polarizable material, it can either be delocalized over a number of symmetrically equivalent sites, or it can become localized at just one of them. In the latter case, the equilibrium positions of the surrounding lattice ions are altered to the extent that a potential well for the charge carrier is formed, in which it becomes strongly bound and immobilized. This self-trapped carrier, together with the surrounding displaced ions, forms a quasiparticle called a small polaron (henceforth referred to simply as a polaron) [1]. Polarons, both in the perfect lattice and bound to defects, are believed to play a central role in the physics and chemistry of many metal oxides, being implicated in phenomena as diverse as photolysis [2], high- $T_{c}$ superconductivity [3], and resistive switching [4]. For this reason, numerous studies have been carried out on technologically relevant oxide materials in which self-trapping is believed to occur [5-16].

Typical preparation procedures of metal oxide samples under ultra-high vacuum lead to the formation of oxygen vacancies. Such defects have long been thought to be the predominant active sites at oxide surfaces, and in the case of the (110) surface of the model oxide rutile $\mathrm{TiO}_{2}$, this has been

Published by the American Physical Society under the terms of the Creative Commons Attribution 3.0 License. Further distribution of this work must maintain attribution to the author(s) and the published article's title, journal citation, and DOI. demonstrated very clearly in recent years [17-20]. The structure of rutile $\mathrm{TiO}_{2}(110)$ is characterized by rows of fivefold coordinated $\mathrm{Ti}$ ions that alternate with rows of bridging oxygen ions. When a bridging oxygen vacancy $\left(\mathrm{O}_{b}\right.$-vac) is formed via the removal of a neutral oxygen containing species (e.g., $\frac{1}{2} \mathrm{O}_{2}$ or $\mathrm{H}_{2} \mathrm{O}$ ), two electrons are left behind in the lattice. While their association with states in the band gap, approximately $0.8 \mathrm{eV}$ below the Fermi level, is well established [21,22], the spatial distributions of these "excess" electrons have been the subject of intense research in recent years, which is still ongoing. The growing consensus from theoretical calculations is that they localize on just two Ti ions close to $\mathrm{O}_{b}$-vacs, reducing the formal charge of the former from +4 to $+3[6,23]$, and may therefore be considered as polarons bound to the $\mathrm{O}_{b}$-vac [24]. Furthermore, theory predicts that the $\mathrm{Ti}^{3+}$ ions reside preferentially in the subsurface layer, although they may also populate the surface layer at higher temperatures $[12,23]$.

On the other hand, the picture provided by the results of scanning tunneling microscopy (STM) experiments presented in the literature is ambiguous: Minato et al. [25] observed a symmetric four-lobe structure of the excess electrons at $78 \mathrm{~K}$, whereas Papageorgiou et al. [8] found an asymmetric two-lobe distribution at $5 \mathrm{~K}$. The reason for this discrepancy is not clear; it could be due to the different temperatures used in the two studies, or it could be that the high density of $\mathrm{O}_{b}$-vacs in the latter study caused the excess electrons to interact with each other [8]. Furthermore, while resonant photoelectron diffraction [26] supports the idea 


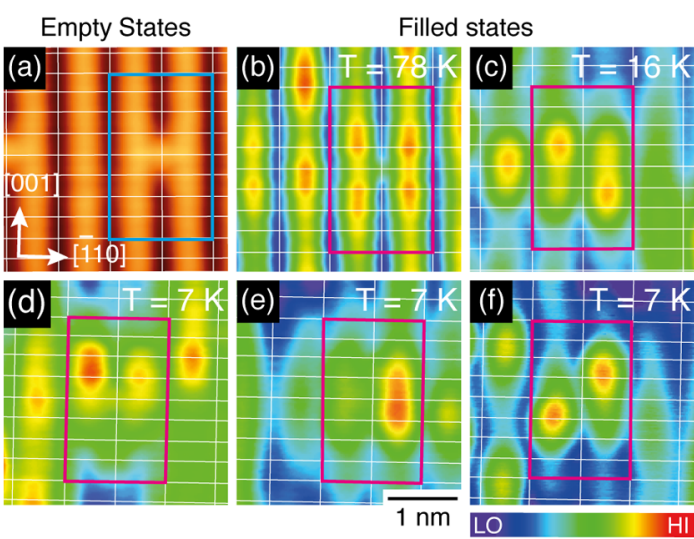

FIG. 1 . $3.2 \times 3.2 \mathrm{~nm}^{2}$ representative STM images of isolated $\mathrm{O}_{b}$-vacs on $\mathrm{TiO}_{2}(110)$ recorded using (a) positive (empty states) and (b)-(f) negative (filled states) sample bias at different temperatures: (a),(b) $78 \mathrm{~K}$, (c) $16 \mathrm{~K}$, and (d)-(f) $7 \mathrm{~K}$. Filled-state images in (b)-(f) were recorded in the vicinity of different isolated $\mathrm{O}_{b}$-vacs. Intersections of the white grids mark the positions of the $\mathrm{O}_{b}{ }^{2-}$. Rectangles are drawn around the $\mathrm{O}_{b}$ vacs and mark the same areas between the empty- and filled-state images in (a),(b). Scan parameters: (a) $\left(V_{S}, I_{T}\right)=+0.9 \mathrm{~V}$, $20 \mathrm{pA}$; (b) $-1.1 \mathrm{~V}, 5 \mathrm{pA}$; (c) $-2 \mathrm{~V}, 1.6 \mathrm{pA}$; and (d)-(f) $-2 \mathrm{~V}, 0.3 \mathrm{pA}$.

that the excess electrons reside at the subsurface, it is not clear that these would be observable in STM experiments.

Here, we employ STM at temperatures between 7 and $78 \mathrm{~K}$ to provide evidence of the polaronic character of the excess electrons associated with $\mathrm{O}_{b}$-vacs on rutile $\mathrm{TiO}_{2}(110)$, and furthermore, to show that they may be manipulated using the STM tip.

Briefly, the experiments were performed using an ultrahigh vacuum, low temperature STM apparatus, configured to take measurements of the current at both positive bias (probing the empty states of the sample) and negative bias (probing the filled states) simultaneously. Values for the negative bias were chosen so as to probe only states within the band gap and not those in the valence band of the material. In order to obtain a sample which is sufficiently conductive at $7 \mathrm{~K}$, yet exhibits $\mathrm{O}_{b}$-vacs which are well separated, we used a special three-step preparation procedure involving the reaction of $\mathrm{O}_{b}$-vacs with water and removal of $\mathrm{H}$ from the resulting bridging hydroxyl groups. No unusual behavior compared to as-prepared surfaces was observed. See the Supplemental Material for further details [27].

Figure 1 shows typical empty- and filled-state STM images of isolated $\mathrm{O}_{b}$-vacs recorded at 78, 16, and $7 \mathrm{~K}$. The empty-state images do not change with temperature, so we focus solely on the filled-state images. At $78 \mathrm{~K}$, all the $\mathrm{O}_{b}$-vacs have an excess electron distribution with a four-lobe structure [Fig. 1(b)]. The lobes are arranged so that the electron distribution exhibits $2 \mathrm{~mm}$ symmetry about the $\mathrm{O}_{b}$-vac, with mirror symmetry about both the [001] and [110] axes, which is consistent with earlier measurements by Minato et al. [25].
At $16 \mathrm{~K}$ [Fig. 1(c)], although the electron distribution around the $\mathrm{O}_{b}$-vac retains a four-lobe structure, two of the lobes appear much brighter, breaking the original mirror symmetry. This symmetry breaking is common to all the $\mathrm{O}_{b}$-vacs that we sampled at $16 \mathrm{~K}$, some having three brighter lobes and some having two, as in Fig. 1(c). The asymmetry in the spatial distribution of the excess electrons is even more pronounced at $7 \mathrm{~K}$. Only two bright lobes are present, and these are oriented either directly across the row [Fig. 1(d)], along the row [Fig. 1(e)], or diagonally across it [Fig. 1(f)]. This asymmetry also appeared in spatially resolved scanning tunneling spectroscopy measurements performed at $5 \mathrm{~K}$ [8], although we emphasize that in that earlier study, interactions between nearby $\mathrm{O}_{b}$-vacs could not be ruled out.

We believe that the temperature dependence of the electronic distribution around the $\mathrm{O}_{b}$-vacs can be understood only if the excess electrons associated with $\mathrm{O}_{b}$-vacs are indeed polarons $[28,29]$. Two of the defining properties of polaronic trapping are the formation of multiple local structural minima associated with their presence at different lattice sites and the fact that their movement occurs via thermally activated hopping; thus, in contrast to charge carriers occupying delocalized states, the diffusion of selftrapped carriers increases with temperature. In the case of a polaron bound to an $\mathrm{O}_{b}$-vac, the polaron is constrained to hop around the vacancy because of its electrostatic interaction with the positively charged vacancy; thus, it is both trapped (at the defect) and self-trapped (at one of a number of equivalent sites) [30].

Previously, an energy barrier of $24 \mathrm{meV}$ was found for electron polaron hopping in rutile $\mathrm{TiO}_{2}$ [31]. Using this value in a simple Arrhenius expression together with an attempt frequency of $10^{13} \mathrm{~Hz}$ (which corresponds to a typical phonon frequency) gives a polaron hopping frequency on the order of $10^{-5} \mathrm{~Hz}$ at $7 \mathrm{~K}$ and $10^{11} \mathrm{~Hz}$ at $78 \mathrm{~K}$. Thus, given that the time scale of the STM scans is on the order of minutes, we would expect the electronic distribution around an isolated $\mathrm{O}_{b}$-vac at $78 \mathrm{~K}$ with a perfectly isotropic environment to be representative of thermal equilibrium. On the other hand, we would expect the distribution observed at $7 \mathrm{~K}$ to arise from kinetic trapping of the polarons in one of a number of symmetrically equivalent, and therefore degenerate, configurations. If this is the case, manipulation of a particular vacancy at $7 \mathrm{~K}$ should lead to a redistribution of the electrons around it, a hypothesis that we now go on to consider.

We use our methodology of precise vacancy manipulation (described in the Supplemental Material [27]) to modify the surface in a way that allows us to explore the dependence of the polaron configuration on the local environment. Figure 2 shows three sequential images recorded during manipulation of a single $\mathrm{O}_{b}$-vac (the full movie is given in Supplemental Movie S1 [27]). It can be seen clearly from the filled-state image in Fig. 2(e) that the 


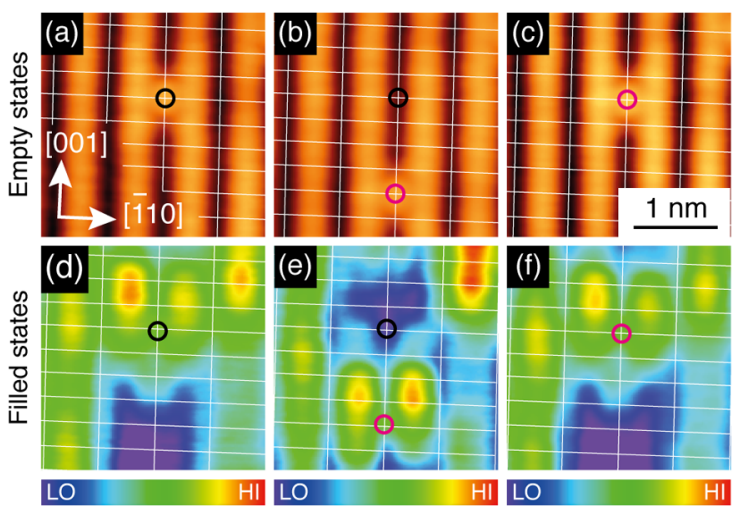

FIG. 2. STM images of an individual $\mathrm{O}_{b}$-vac of $\mathrm{TiO}_{2}(110)$ taken (a),(d) before manipulation, (b),(e) after the $\mathrm{O}_{b}$-vac was displaced along the $\mathrm{O}_{b}$ row by a series of $+4 \mathrm{~V}, 6 \mathrm{~s}$ tip pulses, and (c),(f) after the $\mathrm{O}_{b}$-vac was returned to its initial position by tip pulses. Black and pink open circles mark the initial and current positions of the $\mathrm{O}_{b}$-vac. Intersections of the white grids mark the positions of $\mathrm{O}_{b}{ }^{2-}$. All images were taken at $7 \mathrm{~K}$. Scan parameters: (a)-(c) $\left(V_{S}, I_{T}\right)=+1.0 \mathrm{~V}, 10 \mathrm{pA}$ and (d)-(f) $-2.0 \mathrm{~V}, 0.3 \mathrm{pA}$.

two lobes have moved from their positions in Fig. 2(d) so that their locations relative to the $\mathrm{O}_{b}$-vac remain unchanged. Our explanation for this is that the observed asymmetric configuration represents the sole ground state. As such, following the manipulation pulses, the polaron distribution relaxes back to this sole ground state rather than being kinetically trapped in one of a number of degenerate ground states. It is well known that oxide surfaces have a nonuniform local potential [32], and we believe that these sorts of variations explain the preferential positioning of the polarons at different locations. To illustrate this point, at $7 \mathrm{~K}$, an energetic preference for a given configuration of just $1 \mathrm{meV}$ would lead to a Boltzmann weight which is approximately 5 times larger than that of the next most stable configuration.

Adding further support to this interpretation, we have also observed reversible changes in the polaron distribution around a vacancy which was manipulated back and forth between two locations. These images are shown in Fig. S2 of the Supplemental Material [27].

As mentioned above, empty-state imaging of $\mathrm{O}_{b}$-vacs is well understood [33-35], but filled-state imaging has received significantly less attention $[8,25,36]$. Thus, the imaging mechanism itself merits some discussion. As shown in Fig. 3(a), our density functional theory calculations indicate that, while the major contribution to the excess electron distribution is from $d_{z^{2}}$ orbitals ( $z$ being normal to the surface) of (formally) $\mathrm{Ti}^{3+}$ ions in the second layer of $\mathrm{Ti}$ atoms, there are also some contributions from $\mathrm{Ti}$ ions at the surface, and it is the electron density decaying into the vacuum above these surface ions that is responsible for the filled-state images. Furthermore, we note that the minor changes in the calculated appearance of the excess electrons upon changes in their proximity to the $\mathrm{O}_{b}$-vac, (a)
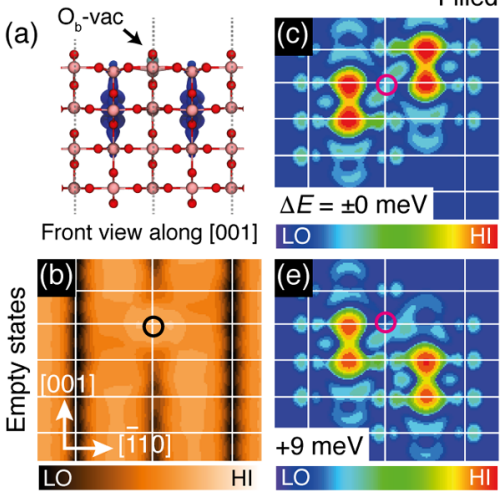

Filled states

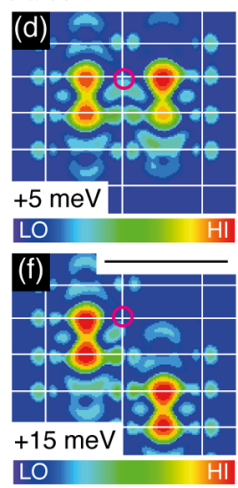

FIG. 3. Calculation of individual $\mathrm{O}_{b}$-vacs on $\mathrm{TiO}_{2}(110)$. (a) Calculated structure of a single $\mathrm{O}_{b}$-vac and one of its ground-state excess electron configurations on rutile $\mathrm{TiO}_{2}(110)$. Turquoise spheres are sites of the removed bridging oxygen ions $\left(\mathrm{O}_{b}{ }^{2-}\right)$. Small pink and red spheres are the Ti and $\mathrm{O}$ ions, respectively. Highlighted in blue is an isosurface of the excess electron density at $10^{-3}$ electrons $/ \AA^{3}$. (b)-(f) Simulated STM images of individual $\mathrm{O}_{b}$-vacs of $\mathrm{TiO}_{2}(110)$ with four nonequivalent polaron configurations. The excess electrons, visualized as red dumbbells in the filled-state images in (c)-(f), are located at different $\mathrm{Ti}$ sites beneath the $\mathrm{O}_{b}$-vacs. Relative energies after ionic relaxation are indicated. Open circles mark the positions of $\mathrm{O}_{b}$-vacs. Intersections of the white grids mark the positions of the $\mathrm{O}_{b}{ }^{2-}$.

also shown in Fig. 3, indicates that their polaronic character in rutile $\mathrm{TiO}_{2}$ is relatively little affected, even when they are subjected to significant perturbations such as the electrostatic field of the $\mathrm{O}_{b}$-vac. Thus, we believe that the excess electrons associated with $\mathrm{O}_{b}$-vacs are representative of both bound and unbound polaronic species, an assertion which has also been made recently by other authors [37].

Notwithstanding the long-ranged potential variations which we believe to be responsible for the preference of a given polaron configuration around an otherwise isolated vacancy, by forming aggregates of vacancies, we now go on to show that we can effectively manipulate the excess electrons by changing their local environments.

By applying a series of $+4 \mathrm{~V}$ pulses to several $\mathrm{O}_{b}$-vacs, we formed $\mathrm{O}_{b}$-vac dimers and trimers (see Supplemental Movies S2 and S3 [27]), as well as a tetramer, at $7 \mathrm{~K}$. In the empty states [Fig. 4(a)], the dimer is characterized by an elliptical protrusion with a spatial extent of two lattice units along the $\mathrm{O}_{b}{ }^{2-}$ row. In the filled states [Fig. 4(b)], the electron distribution appears to comprise four bright lobes and a central spot appearing less bright than the lobes. The four bright lobes, which are located on the nearest $\mathrm{Ti}_{5 c}$ neighbors to the $\mathrm{O}_{b}$-vac dimer, are of unequal brightness, consistent with earlier measurements [36] where $\mathrm{O}_{b}$-vac dimers were formed in an uncontrolled way during a $+3 \mathrm{~V}$ scan on the $\mathrm{TiO}_{2}(110)$ surface at temperatures of $80 \mathrm{~K}$ and above. This symmetry breaking once again is indicative of the polaronic nature of the excess electrons in contrast with 


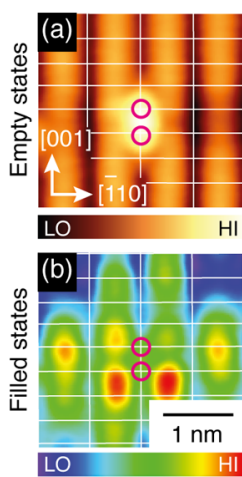

Dimer
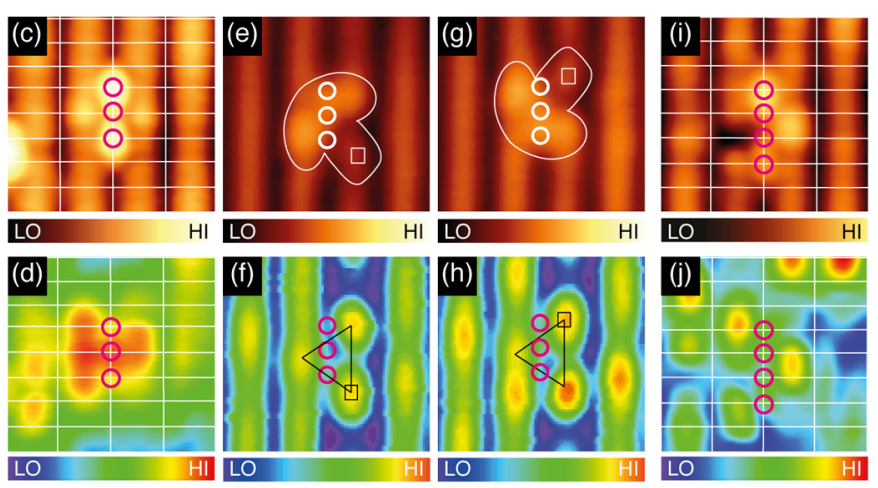

'cross-dumbbell'

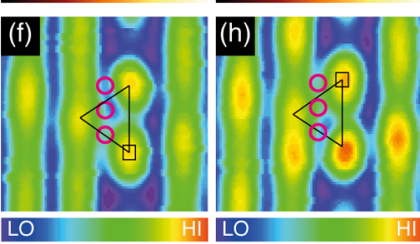

'mushroom' trimers

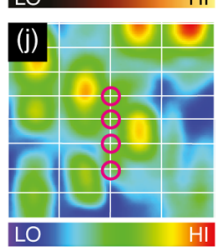

Tetramer

trimer

FIG. 4. Experimental STM images of $\mathrm{O}_{b}$-vac multimers obtained simultaneously at positive and negative sample biases. The $\mathrm{O}_{b}$-vac dimer in (a) and (b), cross-dumbbell trimer in (c) and (d), and tetramer in (i) and (j) were formed using a series of $+4 \mathrm{~V}, 6 \mathrm{~s}$ tip pulses at $7 \mathrm{~K}$. The mushroom trimers in (e)-(h) were formed by applying $+3.75 \mathrm{~V}, 0.2 \mathrm{~s}$ tip pulses over a cross-dumbbell trimer at $78 \mathrm{~K}$. The intersections of the white grids mark the positions of $\mathrm{O}_{b}{ }^{2-}$. Circles mark the removed $\mathrm{O}_{b}{ }^{2-}$. Scan parameters: (a), (c), (i) $\left(V_{S}, I_{T}\right)=+1.0 \mathrm{~V}, 10 \mathrm{pA},(\mathrm{b}),(\mathrm{d}),(\mathrm{j})-2.0 \mathrm{~V}, 0.3 \mathrm{pA}$, (e), (g) $+0.7 \mathrm{~V}, 10 \mathrm{pA}$, and (f),(h) $-1.0 \mathrm{~V}, 1.6 \mathrm{pA}$. STM images in (a)-(d) and (i) and (j) were taken at $7 \mathrm{~K}$, those in (e)-(h) taken at $78 \mathrm{~K}$.

the symmetric, delocalized description of the density functional theory calculations of Cui et al. [36].

Figures 4(c) and 4(d) show an $\mathrm{O}_{b}$-vac trimer at $7 \mathrm{~K}$. In the empty-state image [Fig. 4(c)], the trimer is characterized by a set of two perpendicularly aligned dumbbell shapes, the brighter of which is parallel to the $\mathrm{O}_{b}$ row. In the filled-state image [Fig. 4(d)], the excess electron distribution is characterized by a dumbbell shape, with the excess electrons mainly populating the nearest $\mathrm{Ti}_{5 c}$ sites and, to a lesser extent, the center of the trimer.

By applying an electrical pulse $(+3.75 \mathrm{~V}, 0.2 \mathrm{~s})$ to a cross-dumbbell trimer [Figs. 4(c) and 4(d)], the structure of the trimer is transformed. In the empty-state image [Fig. 4(e)], the trimer is now characterized by a mushroom shape: it has a stem consisting of a dark, round feature that resides at the bottom-right corner and a bright cap comprising an elliptical part and a round part that are located at the top-right and bottom-left corners of the trimer, respectively. In the filled-state image [Fig. 4(f)], it has a three-lobe structure with a triangular configuration.

We examine whether or not this transformation is reversible by applying additional electrical pulses to the mushroom trimer in Figs. 4(e) and 4(f). Following an electrical pulse, the mushroom transforms into a structure which is equivalent but reflected across the [1110] axis, as shown in Figs. 4(g) and 4(h). Another pulse causes the trimer to revert to the original cross-dumbbell shape. We emphasize that each transformation is accompanied by a corresponding change in the excess electron distribution; thus, pulsing of the trimer alters the distribution of the excess electrons in a controlled and reversible way. Simulated empty- and filled-state STM images for the $\mathrm{O}_{b}$-vac dimer, two types of trimers, and a tetramer are shown in Supplemental Fig. S3 [27].

Our simulations show that when a cross-dumbbell trimer transforms into the mushroom structure, one of the two most undercoordinated Ti ions at the center of the vacancy complex is relocated to a subsurface octahedral interstitial site, increasing its coordination number from 4 to 6 . Each of the two Ti ions can relocate to the left or right of their original positions, accounting for the reflection symmetries observed in the experiments. This gives a total of five structural configurations: four mushrooms and one cross dumbbell (one of the mushroom configurations is illustrated in Supplemental Fig. S3 [27]). According to our calculations, the electrons are redistributed around the vacancy complex following the structural changes but remain well localized in all the configurations considered; thus, in effect, the polarons are manipulated by the STM tip via the induced structural changes.

While the cross-dumbbell and mushroom configurations are essentially equal in energy to within the accuracy of the calculations, the energy barrier for the transformation between the two structures was calculated to be $\sim 0.3 \mathrm{eV}$, which should prevent thermal conversion at the low temperatures used in our experiments. This is consistent with the fact that we do not observe any change to the structure of a trimer without applying a tip pulse.

Finally, we note that the susceptibility of the vacancy complexes to such distortions appears to be a fairly general feature. The nonlinear appearance of the tetramer in the experiment [Figs. 4(i) and 4(j)] is due to a similar displacement of a $\mathrm{Ti}$ ion that enables the system to gain $\sim 0.5 \mathrm{eV}$ in energy (see Supplemental Fig. S3 [27]).

To summarize, we have shown that the excess electrons associated with $\mathrm{O}_{b}$-vacs on $\mathrm{TiO}_{2}(110)$ are polaronic in nature, but that their precise distribution around isolated vacancies at low temperatures is governed by electric fields in the surface. However, their distribution can be controlled by manipulating the $\mathrm{O}_{b}$-vacs into complexes of several adjacent vacancies. We have shown that the electrons 
around a vacancy trimer can be made to adopt a number of configurations, which can be selected by an STM tip pulse. Thus, we demonstrate that it is possible to engineer the configurations of polarons, which allows for the study of their interactions and which may allow the construction of directional conduction pathways. This could be important, for example, in memristive devices.

We acknowledge useful discussions with Professor Alex Shluger. This work was supported by the European Research Council Advanced Grant ENERGYSURF (G. T.), the Engineering and Physical Sciences Research Council (EPSRC) (UK), European Cooperation in Science and Technology Action CM1104, the Royal Society (UK), and Alexander von Humboldt Stiftung (Germany). M. J. W. and K. H. acknowledge funding from the Swedish Research Council (VR) and the Swedish Foundation for International Cooperation in Research and Higher Education (STINT). Through our membership of the UK's High Performance Computing Materials Chemistry Consortium, which is funded by the EPSRC (EP/L000202), this work made use of the facilities of High-End Computing Terascale Resource (HECToR) and Advanced Research Computing High-End Resource (ARCHER), the UK's national highperformance computing service, which is funded through the EPSRC's High End Computing Programme.

*Corresponding author. g.thornton@ucl.ac.uk

[1] D. Emin, Polarons (Cambridge University Press, New York, 2013).

[2] S. D. Jackson and J. S. J. Hargreaves, Metal Oxide Catalysis (Wiley, Weinheim, 2008).

[3] E. Salje, A. S. Alexandrov, and W. Y. Liang, Polarons and Bipolarons in High-Tc Superconductors and Related Materials (Cambridge University Press, Cambridge, England, 2005).

[4] M. Wang, C. Bi, L. Li, S. Long, Q. Liu, H. Lv, N. Lu, P. Sun, and M. Liu, Nat. Commun. 5, 4598 (2014).

[5] P. Deák, B. Aradi, and T. Frauenheim, Phys. Rev. B 86, 195206 (2012).

[6] M. Setvin, C. Franchini, X. Hao, M. Schmid, A. Janotti, M. Kaltak, C. G. Van de Walle, G. Kresse, and U. Diebold, Phys. Rev. Lett. 113, 086402 (2014).

[7] S. Moser, L. Moreschini, J. Jaćimović, O. S. Barišić, H. Berger, A. Magrez, Y. J. Chang, K. S. Kim, A. Bostwick, E. Rotenberg, L. Forró, and M. Grioni, Phys. Rev. Lett. 110, 196403 (2013).

[8] A. C. Papageorgiou, N. S. Beglitis, C. L. Pang, G. Teobaldi, G. Cabailh, Q. Chen, A. J. Fisher, W. A. Hofer, and G. Thornton, Proc. Natl. Acad. Sci. U.S.A. 107, 2391 (2010).

[9] C. Di Valentin, G. Pacchioni, and A. Selloni, Phys. Rev. Lett. 97, 166803 (2006).

[10] J.-F. Jerratsch, X. Shao, N. Nilius, H.-J. Freund, C. Popa, M. V. Ganduglia-Pirovano, A. M. Burow, and J. Sauer, Phys. Rev. Lett. 106, 246801 (2011).
[11] K. P. McKenna, M. J. Wolf, A. L. Shluger, S. Lany, and A. Zunger, Phys. Rev. Lett. 108, 116403 (2012).

[12] H. M. Rønnow, C. Renner, G. Aeppli, T. Kimura, and Y. Tokura, Nature (London) 440, 1025 (2006).

[13] S. Chrétien and H. Metiu, J. Phys. Chem. C 115, 4696 (2011).

[14] N. A. Deskins and M. Dupuis, Phys. Rev. B 75, 195212 (2007).

[15] H. Sezen, M. Buchholz, A. Nefedov, C. Natzeck, S. Heissler, C. Di Valentin, and C. Wöll, Sci. Rep. 4, 3808 (2014).

[16] M. Setvin, M. Schmid, and U. Diebold, Phys. Rev. B 91, 195403 (2015).

[17] S. Wendt, R. Schaub, J. Matthiesen, E. K. Vestergaard, E. Wahlström, M. D. Rasmussen, P. Thostrup, L. M. Molina, E. Lægsgaard, I. Stensgaard, B. Hammer, and F. Besenbacher, Surf. Sci. 598, 226 (2005).

[18] O. Bikondoa, C. L. Pang, R. Ithnin, C. A. Muryn, H. Onishi, and G. Thornton, Nat. Mater. 5, 189 (2006).

[19] Z. Zhang, O. Bondarchuk, B. D. Kay, J. M. White, and Z. Dohnálek, J. Phys. Chem. B 110, 21840 (2006).

[20] D. Matthey, J. G. Wang, S. Wendt, J. Matthiesen, R. Schaub, E. Lægsgaard, B. Hammer, and F. Besenbacher, Science 315, 1692 (2007).

[21] V. E. Henrich, G. Dresselhaus, and H. J. Zeiger, Phys. Rev. Lett. 36, 1335 (1976).

[22] R. L. Kurtz, R. Stock-Bauer, T. E. Madey, E. Román, and J. L. De Segovia, Surf. Sci. 218, 178 (1989).

[23] P. M. Kowalski, M. F. Camellone, N. N. Nair, B. Meyer, and D. Marx, Phys. Rev. Lett. 105, 146405 (2010).

[24] A. L. Shluger, K. P. McKenna, P. V. Sushko, D. M. Ramo, and A. V. Kimmel, Model. Simul. Mater. Sci. Eng. 17, 084004 (2009).

[25] T. Minato, Y. Sainoo, Y. Kim, H. S. Kato, K.-I. Aika, M. Kawai, J. Zhao, H. Petek, T. Huang, W. He, B. Wang, Z. Wang, Y. Zhao, J. Yang, and J. G. Hou, J. Chem. Phys. 130, 124502 (2009).

[26] P. Krüger, J. Jupille, S. Bourgeois, B. Domenichini, A. Verdini, L. Floreano, and A. Morgante, Phys. Rev. Lett. 108, 126803 (2012).

[27] See Supplemental Material at http://link.aps.org/ supplemental/10.1103/PhysRevLett.117.116402 for experimental details, calculation details, and supplemental figures and movies.

[28] N. A. Deskins, R. Rousseau, and M. Dupuis, J. Phys. Chem. C 115, 7562 (2011).

[29] T. Shibuya, K. Yasuoka, S. Mirbt, and B. Sanyal, J. Phys. Chem. C 118, 9429 (2014).

[30] A. M. Stoneham, J. Gavartin, A. L. Shluger, A. V. Kimmel, D. M. Ramo, H. M. Rønnow, G. Aeppli, and C. Renner, J. Phys. Condens. Matter 19, 255208 (2007).

[31] S. Yang, A. T. Brant, N. C. Giles, and L. E. Halliburton, Phys. Rev. B 87, 125201 (2013).

[32] C. Barth and C. R. Henry, Nanotechnology 17, S155 (2006).

[33] C. L. Pang, R. Lindsay, and G. Thornton, Chem. Rev. 113, 3887 (2013).

[34] U. Diebold, Surf. Sci. Rep. 48, 53 (2003).

[35] M. A. Henderson, Surf. Sci. Rep. 66, 185 (2011).

[36] X. Cui, B. Wang, Z. Wang, T. Huang, Y. Zhao, J. Yang, and J. G. Hou, J. Chem. Phys. 129, 044703 (2008).

[37] P. G. Moses, A. Janotti, C. Franchini, G. Kresse, and C. G. Van de Walle, J. Appl. Phys. 119, 181503 (2016). 